\title{
Contextualizing Al Education for K-12 Students to Enhance Their Learning of Al Literacy Through Culturally Responsive Approaches
}

\author{
Amy Eguchi ${ }^{1}\left[\right.$ [ Hiroyuki Okada ${ }^{2} \cdot$ Yumiko Muto ${ }^{3}$
}

Received: 26 July 2020 / Accepted: 14 June 2021 / Published online: 6 August 2021

๑) Gesellschaft für Informatik e.V. and Springer-Verlag GmbH Germany, part of Springer Nature 2021

\begin{abstract}
$\mathrm{AI}$ has become ubiquitous in our society, accelerated by the speed of the development of machine learning algorithms and voice and facial recognition technologies used in our everyday lives. Furthermore, AI-enhanced technologies and tools are no strangers in the field of education. It is more evident that it is important to prepare K-12 population of students for their future professions as well as citizens capable of understanding and utilizing AI-enhanced technologies in the future. In response to such needs, the authors started a collaborative project aiming to provide a K-12 AI curriculum for Japanese students. However, the authors soon realized that it is important to contextualize the learning experience for the targeted K-12 students. The paper aims at introducing the idea of contextualizing AI education and learning experience of K-12 students with examples and tips using the work-in-progress version of the contextualized curriculum using culturally responsive approaches to promote the awareness and understanding of AI ethics among middle school students.
\end{abstract}

Keywords Contextualization $\cdot$ AI literacy $\cdot$ Culturally responsive pedagogy $\cdot$ K-12 AI education $\cdot$ Cultural context

\section{Introduction}

In 2018, Forbes [1] published a blog post stating:

Over the next 5 years, we are about to witness the world we live in entirely disrupted by improvements in artificial intelligence (AI) and machine learning. (para. 1)

AI's influence has already reached all parts of society where we have available data and/or improvement is needed

Amy Eguchi

a2eguchi@ucsd.edu

Hiroyuki Okada

h.okada@eng.tamagawa.ac.jp

Yumiko Muto

muto@lab.tamagawa.ac.jp

1 Department of Education Studies, University of California San Diego, 9500 Gilman Drive \#0070, La Jolla, CA 92093-0070, USA

2 Brain Science Institute, Graduate School of Engineering, Graduate School of Brain Sciences, Tamagawa University, 6-1-1 Tamagawagakuen, Machida, Tokyo 194-8610, Japan

3 Brain Research institute, Tamagawa University, 6-1-1 Tamagawagakuen, Machida, Tokyo 194-8610, Japan either by automation or inventions [3]. The rapid availability and development of computer systems capable of processing a large amount of data faster and more accurately than humans can have contributed to the integration of AI in our lives. In other words, human activities in society have already been impacted or can be impacted by AI, sooner or later. AI was a thing in science fiction for many decades before becoming part of our everyday lives. The concept of AI was introduced in Alan Turing's concept of "thinking machines" in the mid-1900s. It is hard to find one uniform definition of AI. IBM Cloud Education [2] offers a clear definition that is easy to understand by the general public:

In computer science, the term artificial intelligence (AI) refers to any human-like intelligence exhibited by a computer, robot, or other machines. In popular usage, artificial intelligence refers to the ability of a computer or machine to mimic the capabilities of the human mind-learning from examples and experience, recognizing objects, understanding and responding to language, making decisions, solving problems-and combining these and other capabilities to perform functions a human might perform, such as greeting a hotel guest or driving a car. (para. 1) 
Children have already been growing up with AI-assistants or AI-assisted smart devices in their homes (i.e. Google enhanced smart speaker, the devices equipped with Siri or Alexa). Rapid development of voice and facial recognition technologies, and machine learning algorithms, which will continue to evolve, have started to transform our everyday lives. It is evident that $\mathrm{AI}$ has become ubiquitous in our society.

The One Hundred Year Study on Artificial Intelligence's Report by the 2015 Study Panel [4] foresees AI to "enhance education at all levels, especially by providing personalization at scale" (p. 31). AI-enhanced interactive machines are already tutoring students in classrooms. It is predicted that the use of these technologies in our lives, including in classrooms, will drastically increase over the next 10 years.

We cannot ignore the fact that the impacts AI have on our society are becoming ever more visible. Educators across fields such as computer science, AI, and education strongly suggest that it is crucial to help people understand the science behind AI, its limits, and its potential societal impacts in the future. It is specifically important and urgent to prepare K-12 students for their future professions as well as citizens capable of understanding and utilizing AI-enhanced technologies in the future [5].

In November 2019, UNESCO organized the Workshop on Teaching and Learning Competencies for Artificial Intelligence (AI) from an Information Access Perspective to examine the elements necessary to support teachers and learners' capacity development for the use of AI. The focus of the discussions during the workshop was the concepts provided by "A Report about Education, Training Teachers and Learning Artificial Intelligence: Overview of Key Issues", the discussion paper commissioned by UNESCO. It identifies five pillars to guide AI for education:

- Uncertainty and Randomness Understanding randomness and accepting uncertainty or the ability to live in a world where models cease to be deterministic;

- Coding and Computational Thinking Skills allowing individuals to create with code and to solve problems through algorithms;

- Data awareness Namely the capacity to build, manipulate, and visualize large amounts of data;

- Critical thinking as adapted to an increasingly Digital society and finally a series of questions amounting to understand our own humanity in view of the changes AI introduces; and

- Post AI Humanism Reconsidering key concepts such as intelligence, experience, creativity, 'the truth' [3].

Responding to the UNESCO's major initiative, several projects and activities have been initiated around the world. For example, Technovation, a global tech education nonprofit and a member of UNESCO's Global Education Coalition, has partnered with UNESCO to organize a free, online 5-week tech education program for girls [6]. During the program, participating girls learn AI, from how it works to how to build their own AI model, in order to create their $\mathrm{AI}$ inventions to solve a program in their community.

The movement to incorporate $\mathrm{AI}$ in education has been observed in different countries in recent years. For example, in April 2018, the first AI textbook for high school students was published in China [7]. The textbook titled "Fundamentals of Artificial Intelligence" was released about six months after China's State Council announced its intention to include AI curriculum in primary and secondary education. China has already published textbooks for both primary and secondary schools while setting its goal to become a world leader in AI by 2030. South Korea has also released its AI strategy aiming to become a top 4 contender in AI by 2022 [8]. It has invested in creating at least six new AI schools to train high-quality Korean engineers. To close the gap in AI talents, it has a focus on building AI capabilities through its education system. In 2017, Singapore's National Research Foundation announced its focus on harnessing the scientific and economic potentials of AI in the country [9]. The National Research Foundation has launched AI Singapore (AISG), a program housed in the National University of Singapore, to realize the goals through AI research, AI technology, AI-innovation, and investments in AI education (talent development). They have developed AI education opportunities for both teachers and students. For example, the AI for Kids (AI4K) program trains schoolteachers and parents via a certified instructor course to become instructors to deliver AI Bootcamp for primary students. EU has also introduced its AI plan in early 2020 [10]. The white paper titled "White Paper on Artificial Intelligence-a European approach to excellence and trust" released by the European Commission has its focus on AI education. It proposes to update the Digital Education Action Plan to increase awareness of AI at all levels of education preparing citizens capable of making informed decisions increasingly affected by AI. In addition, it plans to develop the revised Coordinated Plan on AI with the Member States to develop the skills necessary to work in AI and developing the workforce for the AI-led transformation. It highlights the strong need and specific efforts to increase the number of women trained and employed in AI-related areas.

In the US, several initiatives at the grassroots level have emerged. AI4ALL (https://ai-4-all.org) is a nonprofit organization, founded in 2017 , dedicated to increasing diversity and inclusion in AI education, research, development, and policy. It has a specific focus on educating underrepresented high school students to become the next generation of AI leaders. It offers summer programs and online open learning for high school students while 
partnering with higher education institutions including Stanford University, University of California-Berkeley, Boston University, Carnegie Mellon University, Princeton University, and Simon Fraser University. AI4K12 (http:// ai4k12.org/) is an initiative jointly sponsored by AAAI and CSTA aiming to develop (1) national guidelines for AI education for K-12, (2) an online, curated resource directory to facilitate AI instruction, and (3) a community of resource and tool developers focused on the AI for K-12 population of students. AI4K12 has released the Five Big Ideas in AI [5] in 2019 which are (Fig. 1):

1. Perception Computers perceive the world using sensors,

2. Representation \& Reasoning Agents maintain representations of the world and use them for reasoning,

3. Learning Computers can learn from data,

4. Natural Interaction Intelligent agents require many kinds of knowledge to interact naturally with humans, and

5. Societal Impact AI can impact society in both positive and negative ways.

This paper focuses on one of the 5 Big Ideas in AI - Societal Impact (ethics) and our attempt to adapt "An Ethics of Artificial Intelligence Curriculum for Middle School Students", AI curriculum focusing on AI ethics developed by Blakeley H. Payne [11] in Japan. It explains, in the process of adapting the curriculum in Japanese educational environment, what modifications were necessary and why.

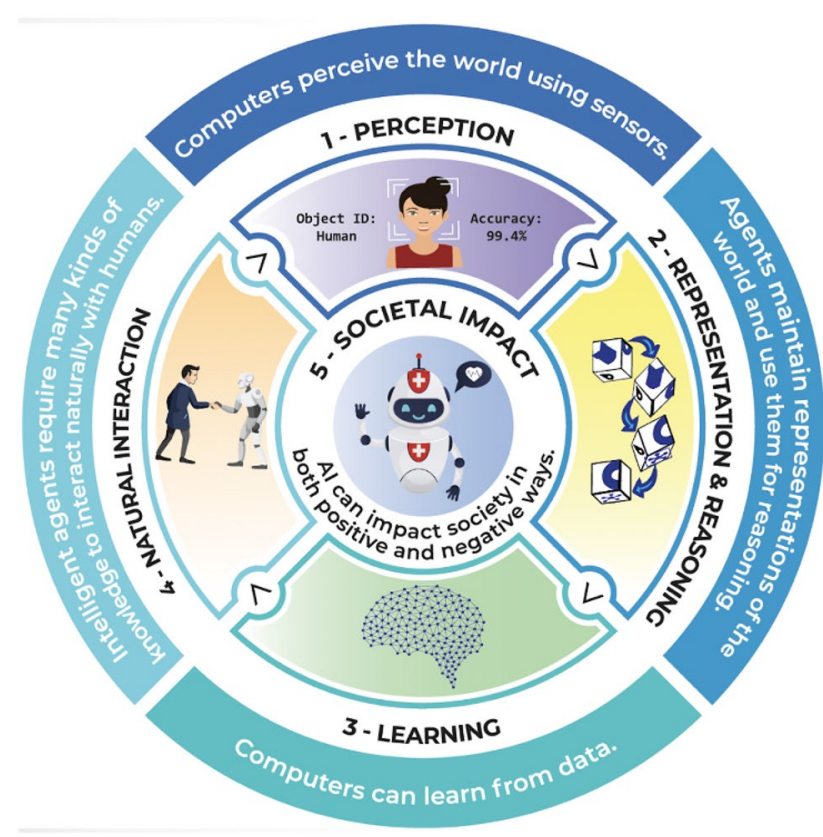

Fig. 15 Big Ideas in $\mathrm{AI}-\mathrm{a}$ guideline to support $\mathrm{AI}$ education in schools

\section{Middle School AI-Ethics Curriculum}

"An Ethics of Artificial Intelligence Curriculum for Middle School Students" (AI-Ethics) developed by Blakeley H. Payne under the direction of Cynthia Breazeal at MIT Media Lab [11] focuses on promoting the understanding of AI ethics in middle school students. It is composed of hands-on, mainly unplugged activities to teach various concepts of AI and its ethical influences, both positive and negative, in society.

"An Ethics of Artificial Intelligence Curriculum for Middle School Students" has five main learning objectives:

1. Understand the basic mechanics of artificial intelligence systems.

2. Understand that all technical systems are socio-technical systems. Understand that socio-technical systems are not neutral sources of information and serve political agendas.

3. Recognize there are many stakeholders in a given sociotechnical system and that the system can affect these stakeholders differently.

4. Apply both technical understanding of AI and knowledge of stakeholders in order to determine a just goal for a socio-technical system.

5. Consider the impact of technology on the world.

The curriculum contains eight activities introducing various AI concepts and ethical issues around AI. "AI Bingo" introduces various AI systems and asks students to identify kind of datasets that each system uses. "Algorithms as Opinions" introduces algorithms as recipes. It then asks students to explore how the best recipe can be defined through which students learn that various opinions are reflected in algorithms. "Ethical Matrix", building on the algorithms as opinions lesson, asks students to identify stakeholders and their values in the algorithms that they created and explore how those values overlap or conflict. "Intro to Supervised Machine Learning \& Algorithmic Bias" introduces students to the concept of classification and machine learning by exploring Google's Teachable Machine tool. "Speculative Fiction" provides opportunities for students to interact with various technologies. After the interactions, it asks students to respond to creative writing prompts about how the technologies might affect people and society. The final three activities on YouTube build on to each other. "YouTube Scavenger Hunt" makes students explore various AI systems on the YouTube platform. Students are tasked to identify what prediction that the algorithm tries to make and what kind of datasets it uses. "YouTube Redesign" asks students to apply what they have learned by constructing an ethical 
matrix around the YouTube Recommender algorithm. The final activity "YouTube Socratic Seminar" involves students in a Socratic seminar discussing about stakeholders influences on YouTube Kids app design.

As part of our project aiming to promote AI literacy in Japanese schools targeting K12 students, "An Ethics of Artificial Intelligence Curriculum for Middle School Students" was selected to be adapted to Japanese classrooms. The following sections explain the project, its rationale, and the process we took to adapt the AI curriculum to be tested in Japanese schools.

\section{Project: Preparing Al-ready Students Through AI for K12 Curriculum and Resources in Japan}

Beginning in 2020, the Japanese national curriculum mandated computer science education offerings starting at elementary schools. However, about $70 \%$ of teachers felt uncertain about the implementation or not ready to implement computer science education in their classrooms. This indicates that there is a need for computer science education curriculum and materials to support classroom teachers. In addition, there are societal needs to promote AI literacy - to prepare the K-12 population of students capable of understanding and utilizing AI-enhanced technologies in the future. Although Japan is one of the leading countries in developing AI-technologies in the world, its educational policy in promoting AI in education, specifically in the $\mathrm{K}-16$ level is lagging behind. Responding to such needs, the authors started a collaborative project aiming to provide a K-16 AI curriculum and resources to promote AI literacy for Japanese students. The project plans to employ the Five Big Ideas in AI in developing the AI curriculum for Japanese students.

The first phase of the project was to identify AI-tools that are suitable for K-12 education. Although the project aims at providing AI literacy in K-16 sequence, initial focus was decided to be K-12 population, while post- 12 level will be explored separately. The AI-tools that visualize how AI works and/or provide a learning environment where students can be a creator of AI-enhanced artifacts or creations and AI-curriculum ideas are collected:

- Cognimates (http://cognimates.me).

- AI for Oceans (https://code.org/oceans).

- Teachable Machine - Google (https://teachablemachine. withgoogle.com/).

- Machine Learning for Kids (https://machinelearningforki ds.co.uk/).

- Quick Draw!-Google (https://quickdraw.withgoogle. com).
- Sketch RNN Demo - Google (https://experiments.withg oogle.com/sketch-rnn-demo).

- MixLab (https://experiments.withgoogle.com/mixlab).

- ecraft2learn (https://ecraft2learn.github.io/ai).

- Gan Dissect (https://gandissect.csail.mit.edu/).

- Speech Notes (https://speechnotes.co/).

- TensorFlow Playground (https://playground.tensorflow. org).

- Unplugged AI (https://classic.csunplugged.org/artificialintelligence).

- Educator's AI Classroom Kit-IBM (https://docs.google. com/document/d/1Zqi74ejYYYLAAEjFBJIYuXUc OMQI56R6K0ZsvQbDmOA/edit).

Next phase is to develop lessons for the curriculum using the AI-tools collected. However, before developing AI-lessons, the authors decided to learn from existing exemplary curriculum. The decision was made to adapt "An Ethics of Artificial Intelligence Curriculum for Middle School Students" to promote the awareness and understanding of AI ethics among middle school students, which is the focus of this paper. The initial intention was to translate and implement the curriculum in a Japanese middle school. Soon after the process was started, the authors realized how simple translation of a well-developed and field-tested curriculum does not work in the new cultural context. It is highlighted in the constructionist learning that educating students out of context will not be relevant or even meaningful for them. It is crucial to make learning authentic and contextualize it in the lives and cultures of students so that it becomes meaningful for them. Especially with the task of teaching AI and ethics, which itself is rather foreign to many of those students, contextualization of the materials and topics used in curriculum help them make sense of the contents covered in the curriculum and enable them to apply newly obtained knowledge in their own lives. To effectively adapt the curriculum in Japanese schools, culturally responsive approaches are used to modify the AI-ethics curriculum to make the activities in the curriculum effective and meaningful for Japanese students. In the following sections, the modifications made with the curriculum are introduced with the rationale behind the modification.

\subsection{Differences in the Use of Al in Society- Al-Enhanced Online Tool}

Although AI-enhanced tools are prominent in our society, how much and what types of AI-enhanced tools are available in the everyday lives of people could vary between countries. For example, Bernard Marr highlighted the 10 best examples of AI tools used in everyday life in the US [12]. The list includes the facial recognition technology to unlock a smartphone, personalization algorithm on social media, 
automatic spell-checkers on Gmail or Goggle Doc to autocollect your writing, digital voice assistances, such as Alexa and Siri, Google Maps and other navigation apps to give you real-time traffic information, and online recommenders on Netflix and Amazon. Although those are the tools that we use every day without even thinking about how AI is contributing to their performances, it does not necessarily mean that those are familiar tools in other countries or cultures.

As many of us are aware, Google tools including Gmail and Google Maps are not available in China. Chinese students may know the names, and some may have used the tools via VPN, those tools could still be foreign to many, especially students in the rural parts of China. The same issue exists in most of Arab countries. However, it does not mean that they have no access to AI-enhanced technologies. In the case of China, Baidu, a Chinese technology company, provides internet-related services including AI-enhanced tools.

When trying to adapt the AI-Ethics curriculum in Japan, the authors encountered a similar issue. Although online services, such as Netflix, Amazon, and YouTube, are also used in Japan, Japanese students are not as familiar with those services as the students in the US. For "YouTube Scavenger Hunt", which tasks students to explore and investigate AItools on YouTube to predict the algorithm that the AI-tool uses and the type of datasets that it needs to make predictions, YouTube was replaced with Yahoo! Japan (https:// www.yahoo.co.jp/), a comprehensive online service providing various news, shopping information, weather information, and more. This is a service widely used by Japanese.

Using the familiar online tools that students can relate to and know how to use will motivate them to engage in the tasks better.

\subsection{Differences in the Use of Al in Society- Al-Enhanced Devices}

There are some AI-enhanced devices used in our everyday lives in the US as mentioned in Marr's article, such as smartphones and smart speakers. iRobot's Roomba has been used in households for almost 20 years. In 2015, iRobot introduced the Roomba 980 which used a new navigation system (vSLAM). From the next generation of Roomba, released in 2018, it can retain the map created. However, in Japan, many more AI-enhanced devices are available in society and they are constantly featured in media including social media. Many new home electronics, such as AC systems, microwaves, and washing machines, come AI capabilities. For example, Sharp, a Japanese electronics company, introduced a microwave with AI-enhanced communication capability. It can respond to you with recommended recipes. Personal/service robotics is another area that has contributed for a broader introduction of AI-technologies in Japanese society. The use of personal/service robots in society is not a special occasion in Japan anymore. Personal/service robots co-existing with people in Japanese society. In Japan, it is not rare to find restaurants and shops where robots are working with people. Robots are serving travelers at airports. SoftBank Robotics' CSR has provided Pepper Robots to public schools since 2017 to support computer science education in schools.

While robots are in the everyday lives of students, if robots or AI-enhanced robotic tools are not part of the curriculum introduced to them, it could be less attractive for them, potentially reducing the level of their engagement in the lessons. In order to make the curriculum more attractive for Japanese students, the authors are exploring options to include activities that involve robots or robotic tools through which students can experience how AI technologies are used and learn AI concepts via their interaction with them.

There are some AI-enhanced robots that can be incorporated in the curriculum such as Zumi (Fig. 2) by Robolink (https://www.robolink.com/zumi/) which has capabilities of vision and navigation.

Micro:Bit (Fig. 3) by BBC (https://www.microbit.org/) is an open-source hardware developed in the UK to support its computing education. Micro:Bit is an all-in-one microcontroller that comes with a variety of sensors and LEDs. It becomes a powerful AI-enhanced robotics tool when combined with a lens, such as HuskyLens by DFRobot. HuskeyLens (Fig.4) is an easy-to-use AI machine vision sensor that can be also connected to Arduino and RaspberryPi (urlhttps://www.dfrobot.com/huskylens.html).
Fig. 2 Zumi, a programmable self-driving car

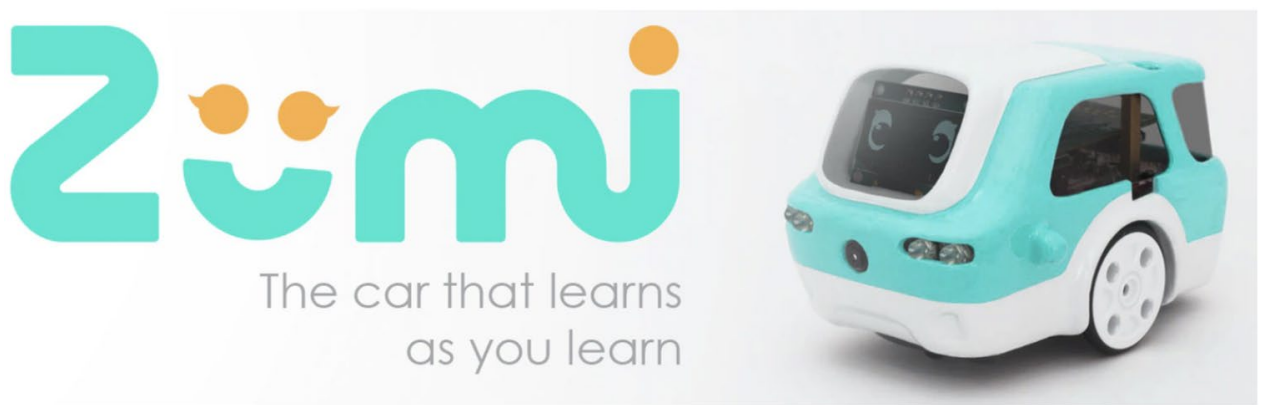




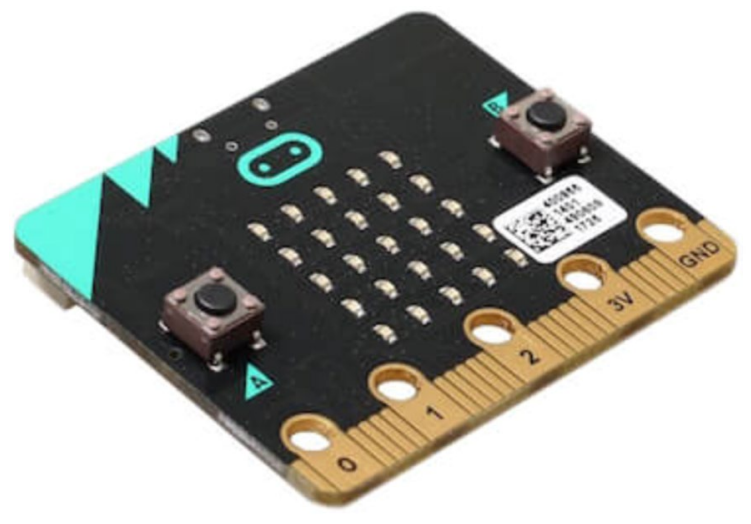

Fig. 3 Micro:Bit - an all-in-one microcontroller for integrating AI

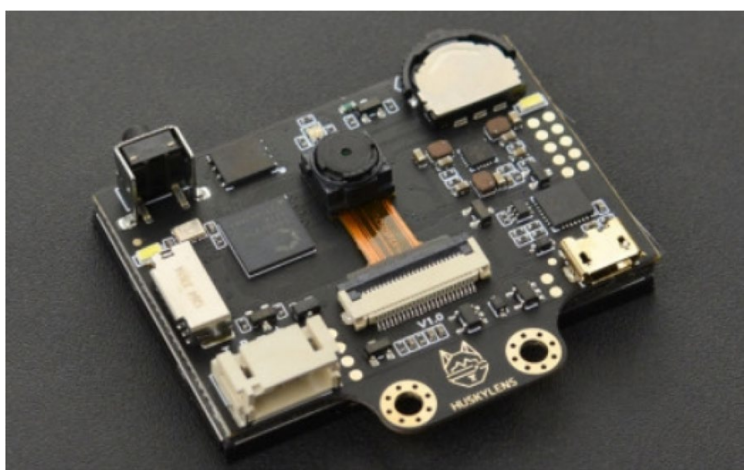

Fig. 4 HuskeyLens-AI machine vision sensor for Micro:Bit

Pepper robot (Fig. 5) by SoftBank Robotics ( https:// www.softbankrobotics.com/us/pepper) is a humanoid robot with AI-capabilities. Pepper is equipped with a range of sensors providing perceptions for it to sense objects and humans around it. Pepper is capable of sensing a touch (three tactile sensors, three bumper sensors, six laser actuators, two sonar sensors, and two IR sensors), hearing (four microphones), and seeing (two RGB cameras and one 3-D sensor). It has a network connectivity through WiFi and Ethernet. Pepper's default perception and interaction capabilities including human detection and tracking, face and voice recognition, gaze detection, and speech capability through its proprietary make it interact rather naturally with humans. Since Pepper can be programmed using Choregraphe (http://doc.aldebaran.com/114/software/choregraphe/choregraphe_overview.html) or block-based programming language, students can program Pepper while learning various AI functions. Since Pepper is available in various schools in Japan, it could be an attractive tool that encourage students to learn AI while engaging with the humanoid.

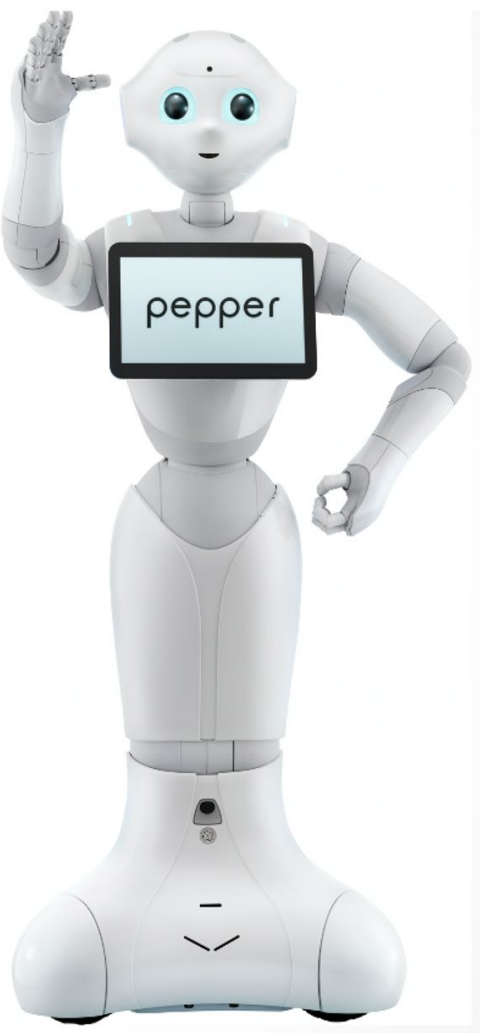

Fig. 5 Pepper-a social robot with multiple AI capabilities

\subsection{Pedagogical Differences}

Pedagogical approaches are an integral part of curriculum contributing to its successful implementation. However, the pedagogical approaches expected could be different between countries, cultures, ethnic groups, regions, school districts, or even schools. Because of the different expectations, it is important to take into account what pedagogical approaches are effective for students in a specific community before making decisions about how to implement a curriculum.

The AI-Ethics curriculum utilizes various active learning pedagogical approaches including hands-on group-/ peer-collaborations, discussions, consensus building, and reflections. Although active learning is becoming a buzz word in the field of education in Japan, the concept is still new to Japanese schoolteachers as well as their students. Historically, the pedagogical approaches in traditional classrooms in Japan are a direct and step-by-step instruction by teachers, which students follow. There are some group-work embedded in the instruction; however, most of the time the instruction is specifically guided or scripted by the teacher with very little explorations by students. A lesson with several group-/peer-collaborations embedded throughout is still very foreign to Japanese classrooms. One of the ultimate goals of the AI literacy project is to promote active learning 
in Japan, which is more effective in terms of students' learning experience and eventually contributes to the students' mastery of the concepts, knowledge, and skills introduced through the curriculum. However, the AI-ethics curriculum is modified to give options as independent work or peer/ group-collaboration allowing teachers to select the best approach for themselves and their students while maintaining its learning goals. Moreover, the modified curriculum keeps the discussion sessions which provide crucial learning opportunities for students without identifying the discussion to be done by a group or the whole class, allowing teachers to decide how to carry out the discussion. In addition, due to the COVID-19 pandemic where remote/online instructions are required, providing independent work as an option allows the curriculum to be easily adapted for remote/online learning environment.

Another modification that was made to accommodate various needs of Japanese teachers and their students was to include the information from the PowerPoint files that the AI-Ethics curriculum provides. With the original curriculum, the ready-to-use PowerPoint files are linked from the main curriculum document, making it easy for teachers to just grab and use them in their classrooms. However, to fully understand the sequence of each lesson, some information from the PowerPoint files needs be inserted into the main curriculum document. It makes it easier for teachers to just read though the main curriculum document to understand what it offers instead of going back and fourth between the PowerPoint files and the main curriculum document. Moreover, the modified file with necessary information from the introduction to a lesson, question prompts for brainstorming/discussions, to the steps of the activity in the lesson, will allow teachers to easily modify the lesson for a remote/ online instruction instead of pulling information out of the PowerPoint files by themselves.

There can be a unique need and/or focus specific to the culture, country, regions, or ethnic group that needs to be incorporated in the education of their children. In 2016, following the United Nation's guideline (https://www.un. org/sustainabledevelopment/), the Japanese government addressed its commitment to United Nation's Sustainable Development Goals (SDCs, Fig. 6). There has been a focus on the SDCs in Japanese education since 2016, and SDCs have been incorporated into various parts of their curriculum. To respond to such a need in Japan, the authors are working to incorporate the SDCs in the AI literacy curriculum.

\section{Discussion-Culturally Responsive Pedagogy for AI Education}

The paper introduces the idea of contextualizing AI education and learning experience of K-12 students with examples and tips using the work-in-progress version of the contextualized curriculum incorporating culturally responsive approaches to promote the awareness and understanding of AI ethics among middle school students in Japan. In this section, why contextualization of the curriculum using culturally responsive approaches is important in AI literacy education will be discussed.

Culturally responsive teaching is a pedagogy designed to motivate students by incorporating teaching practices grounded in their experiences and ways in which they make sense of their knowledge [13-17]. It places students' cultural identities at the center of their learning process while
Fig. 6 United Nation's Sustainable Development Goals - a collection of 17 interlinked global goals as a "blueprint to achieve a better and more sustainable future for all"

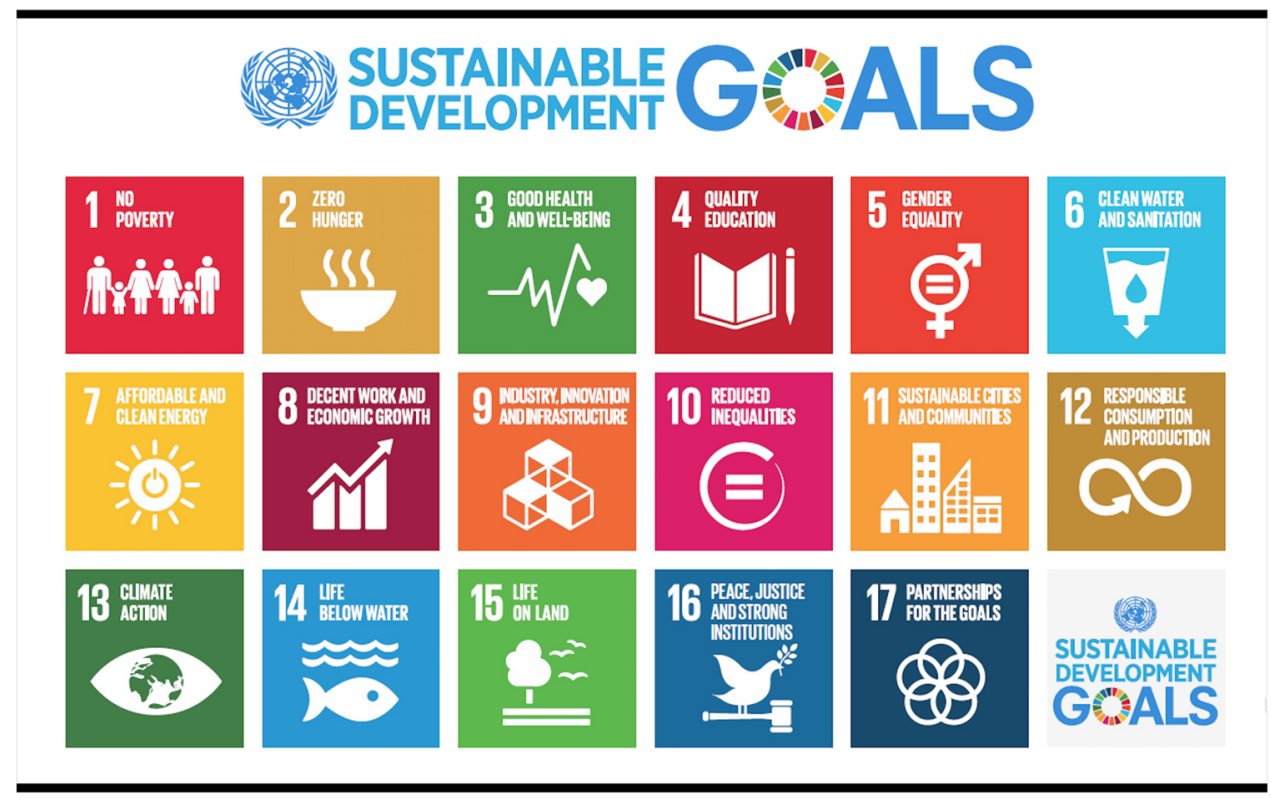


incorporating various aspects of their lives including their cultural knowledge, prior experience and knowledge, ways of thinking into the curriculum. Its focus is to facilitate and support all students with their learning journey by making it relevant and relatable for the learners [18]. Through culturally responsive approaches, students learn effectively in a culturally supported, learner-centered learning environment, while the strengths students bring into the classroom are recognized, nurtured, and enhanced to promote students' achievement. The topics and approaches to learning are contextualized, making connections to the learners' everyday lives. One of the dimensions of culturally responsive pedagogy is instructional which includes materials, strategies, and activities that shape the foundation of instruction. Gay explains that any instruction should be influenced by culture and cultural characteristics of their students informing teachers how to modify their instructional strategies to address the diversity of their students [14]. Ladson-Billings emphasizes that culturally responsive pedagogy helps marginalized population of students to improve their self-concept and self-image as well as cultural competence [16]. Moreover, it fosters their ability to critically evaluate social order, which in turn helps them to become academically successful and socially conscious citizen.

Culturally responsive computing education is not new anymore [19-24]. It is stated that culturally responsive pedagogy "can be used to explore problems and solutions in any scientific or technical field, often using traditional knowledge or practices of the group being educated" [19]. Eglash et al. introduced an example of a student from Tanzania studying at the University of Finland not understanding programming examples that referenced European games of chance to explain that culturally responsive pedagogy can be incorporated anywhere to benefit students who do not come from the group that created the curriculum. It is also pointed out that a wide variety of culturally responsive frameworks in computing education, also known as "ethnocomputing", have already been developed [19]. Miller who uses hip-hop in computing education emphasizes that integrating hiphop which is the language of the African-American youth into computer science to unearth the lyrical complexity of hip-hop allows the underrepresented group of students to understand how their culture has relevance in computer science and provides the reason for them to engage deeper into the world of computer science [24]. As Miller points out by referring to Papert's theory of constructionism, new knowledge is built upon previously constructed knowledge and the schema each student has already acquired. Incorporating culturally responsive approaches in curriculum ensures that there is cultural resonance - making connections between the knowledge and experience that students have previously constructed and what they will explore in order to construct new knowledge through their experience.
Culturally responsive teaching incorporates diverse ethnic individuals and groups; contextualize issues within (and across) race, class, ethnicity, and gender; and includes multiple kinds of knowledge and perspectives [14]. The integration of culturally responsive pedagogy into AI literacy education is specifically relevant because $\mathrm{AI}$ itself is new to almost everyone including teachers and parents. Not many teachers and parents fully understand how AI is perceived, viewed, accepted (or not), or has influenced their students' lives. Introducing AI literacy to diverse learners successfully requires making $\mathrm{AI}$ and its concepts relevant and relatable by using culturally responsive pedagogy. This approach helps students see the connection between AI and what they are interested in and familiar with, as AfricanAmerican students could between hip-hop and computing education, which will deeply engage them in the new learning experience.

\section{Conclusion}

It was two decades ago, Hendler [25] expressed his observation about robotics evolution:

A funny thing is happening in the field of robotics. A revolution is occurring without being noticed by many in the robotics research community. The robotics journals and conferences have largely missed the fact that robots are starting to leave the laboratory and make it out into the world. (p. 2)

Now, this is happening with AI as technological-tools and content for learning from possibly pre-kindergarten to postsecondary students. However, the degree and types of AI integration in society varies from country to country, or culture to culture. This is the topic of discussions in this paper. It introduced how AI has been integrated in and influenced our lives, and how the issues around introducing $\mathrm{AI}$ in $\mathrm{K}-12$ education in various countries have been addressed. It, then, discussed how the integration of AI literacy in education is lagged behind in Japan, which has just laid out its computer science education. The project discussed in the paper aiming at introducing K-16 education in Japan. The paper highlighted the importance of creating culturally responsive AI literacy using the project's approach to incorporating culturally responsive approaches in a well-developed and field-tested AI-Ethics curriculum.

As Papert [26] argues, children construct new knowledge upon previously constructed knowledge and the schema they have already acquired. Taking culturally responsive pedagogy into account when developing an AI literacy curriculum or lessons is a crucial approach to ensure the materials and teaching strategies integrated are relevant and meaningful for the learners. By doing so, we connect AI literacy and/ or AI technologies and tools introduced with the learners' 
cultures and everyday lives, which in return engage them and promote their interests in learning about the field of AI.

Although the project is still at the beginning stage and more work needs to be done to make the K-16 AI literacy education meaningful, relevant and relatable for Japanese learners, ensuring the development of culturally responsive AI literacy for Japanese audience from the beginning will contribute to its success in the near future.

\section{References}

1. Delgado A (2018) three impacts of artificial intelligence on society. Forbes-Innovation, Jun. 13, https://www.forbes.com/sites/ forbestechcouncil/2018/06/13/three-impacts-of-artificial-intel ligence-on-society/\#300acc266ec0. Accessed 3 Jun 2020

2. IBM Cloud Education (2020) Artificial intelligence. IBM Cloud Learn Hub. Jun. 03. https://www.ibm.com/cloud/learn/what-isartificial-intelligence. Accessed $20 \mathrm{Feb} 2021$

3. Higuera CD (2019) A report about education, training teachers and learning artificial intelligence: overview of key issues

4. 2015 Study Panel-One Hundred Year Study on Artificial Intelligence (2016) Artificial intelligence and life in 2030. Stanford University

5. Touretzky D, Martin F, Gardner-McCune C Seehorn D (2019) Envisioning AI for K-12: what should every child know about AI? In: Thirty-Third AAAI Conference on artificial intelligence, Honolulu, Hawaii, vol. 33. https://www.semanticscholar.org/ paper/Envisioning-AI-for-K-12\%3A-What-Should-Every-ChildAI-Touretzky-Gardner-McCune/ceb31a1f6901a7aa5995af472 abaac $1035 \mathrm{f} 3 \mathrm{a} 154$

6. UNESCO (2020) "UNESCO joins Technovation to launch free, online, 5-week tech education programme for girls in 6 countries," Jul. 17. https://n.unesco.org/news/ unesco-joins-technovati on-launch-free-online-5-week-tech-education-programme-girls-6countries. Accessed 21 July 2020

7. Jing M (2018) "China looks to school kids to win the global AI race," South China Morning Pots, May 03. https://www.scmp. com/tech/china-tech/article/2144396/china-looks-school-kidswin-global-ai-race. Accessed 21 July 2020

8. Walch K (2018) "Is South Korea poised to be a leader in AI?," Forbes, Sep. 07. https://www.forbes.com/sites/cognitiveworld/ 2018/09/07/is-south-korea-poised-to-be-a-leader-in-ai/\#12d0c d47fa2f. Accessed 21 July 2020

9. Koo S, Liew L (2020) Intergenerational Learning with AI for Kids (AI4K)TM. In: Presented at the International Workshop on education in artificial intelligence K-12 (EduAI-20)

10. European Commission (2020) "White paper on artificial intelligence-a European approach to excellence and trust," $\operatorname{COM}(2020) 65$ final, Jul. [Online]. https://ec.europa.eu/commi ssion/sites/beta-political/files/political-guidelines-next-commi ssion_en.pdf, Acceced May 30, 2020. Acceced 30 May 2020

11. Payne BH (2019) An ethics of artificial intelligence curriculum for middle school students. MIT Media Lab-AI Education. https:// aieducation.mit.edu/aiethics.html. Accessed 30 May 2020
12. Marr B (2019) "The 10 best examples of how AI is already used in our everyday life," Forbes-Innovation. Dec. 16. https://www. forbes.com/sites/bernardmarr/2019/12/16/the-10-best-examplesof-how-ai-is-already-used-in-our-everyday-life/\#60b63df81171. Accessed 10 July 2020

13. Rhodes CM, Schmidt SW (2018) Culturally responsive teaching in the online classroom. eLearn Magazine

14. Gay G (2002) Preparing for culturally responsive teaching. J Teach Educ 53(2):106-116

15. Gay G (2018) Culturally responsive teaching theory, research, and practice, 3rd edn. Teachers College Press, New York

16. Ladson-Billings G (1995) Toward a theory of culturally responsive pedagogy. Am Educ Res J 32(3):465-491

17. Ladson-Billings $G$ (2014) Culturally relevant pedagogy 2.0: a.k.a. the Remix. Harv Educ Rev 84(1):74-84

18. Richards HV, Brown AF, Forde TB (2007) Addressing diversity in schools: culturally responsive pedagogy. TEACHING Except Child 39(3):64-68

19. Eglash R, Gilbert JE, Foster E (2013) Broadening participation toward culturally responsive computing education-improving academic success and social development by merging computational thinking with cultural practices. Commun ACM 7(56):33-36

20. Scott KA, Sheridan KM, Clark K (2015) Culturally responsive computing: a theory revisited. Learn Media Technol 40(4):412-436

21. Kafai YB, Searle K, Martinez C, Brayboy B (2014) Ethnocomputing with electronic textiles: culturally responsive open design to broaden participation in computing in American Indian youth and communities. In SIGCSE 2014 - Proceedings of the 45th ACM Technical Symposium on Computer Science Education. Association for Computing Machinery pp 241-246. https://doi.org/10. $1145 / 2538862.2538903$

22. Webb A, Gonzales R (2018) Striving for Excellence in Introductory Computer Science Courses Using Culturally Responsive Teaching (CRT). in SIGCSE 2018: 408-409. https://doi.org/10. $1145 / 3159450.3159631$

23. Codding D, Mouza C, Rolón-Dow R, Pollock L (2019) Positionality and belonging: analyzing an informally situated and culturally responsive computer science program. In: FL2019: Proceedings of FabLearn 2019, March 2019 pp 132-135. https://doi.org/10. $1145 / 3311890.3311909$

24. Miller O (2014) It's deeper than rap, toward culturally responsive CS-using hip-hop lyrics and artificial intelligence to engage more students in computer science based on their cultural background. XROS 20(4):28-30

25. Hendler J (2000) Robots for the rest of us: designing systems 'Out of the box'. In: Druin A, Hendler J (eds) Robots for kids: exploring new technologies for learning. Academic Press, San Diego pp 2-7

26. Papert S (1993) Mindstorms: children, computers, and powerful ideas (2nd edition), 2nd edn. Basic Books, New York 